\title{
Primary Health Care
}

\section{Research \& \\ Development}

SAPC Annual Conference 2010 Abstracts, 7-9 July 2010, University of East Anglia, UK

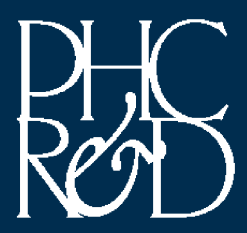

